Proceedings of the Edinburgh Mathematical Society (2007) 50, 411-428 (C)

DOI:10.1017/S0013091505000428 Printed in the United Kingdom

\title{
DIMENSIONS OF INTERSECTIONS OF THE SIERPINSKI CARPET WITH LINES OF RATIONAL SLOPES
}

\author{
QING-HUI LIU ${ }^{1}$, LI-FENG XI ${ }^{2}$ AND YAN-FEN ZHAO ${ }^{3}$ \\ ${ }^{1}$ Department of Computer Science and Engineering, Beijing Institute of Technology, \\ 100080, Beijing, People's Republic of China (qhliu@bit.edu.cn) \\ ${ }^{2}$ Institute of Mathematics, Zhejiang Wanli University, Ningbo, 315100, \\ Zhejiang, People's Republic of China (xilifengningbo@yahoo.com) \\ ${ }^{3}$ Department of Mathematics, Wuhan University, Wuhan, 430062, Hubei, \\ People's Republic of China (wangzhaoyanfen@hotmail.com)
}

(Received 30 March 2005)

\begin{abstract}
This paper computes the Box and Hausdorff dimensions of the intersections of the Sierpinski carpet with planar lines of rational slopes.
\end{abstract}

Keywords: fractal; Sierpinski carpet; dimension; ergodic theory

2000 Mathematics subject classification: Primary 28A80

\section{Introduction}

For eight points $\left(x_{i}, y_{i}\right) \in\{0,1,2\} \times\{0,1,2\} \backslash\{(1,1)\}$, let $\phi_{i}(x, y)=\frac{1}{3}(x, y)+\frac{1}{3}\left(x_{i}, y_{i}\right)$. Then the Sierpinski carpet $F$ of $\mathbb{R}^{2}$ is the invariant set of $\left\{\phi_{i}\right\}_{i=1}^{8}$ with $\operatorname{dim}_{\mathrm{H}} F=$ $\operatorname{dim}_{\mathrm{B}} F=\log 8 / \log 3[\mathbf{4}]$.

Given $\theta \in[0,2 \pi) \backslash\{\pi / 2,3 \pi / 2\}$, let $L_{\theta, a}$ be the line $y=(\tan \theta) x+a$, and the section $F_{\theta, a}=L_{\theta, a} \cap F$, the intersection of the Sierpinski carpet and the planar line. For any $\theta \neq \pi / 2,3 \pi / 2$, the interval $J_{\theta}$ is defined by

$$
J_{\theta}= \begin{cases}{[-\tan \theta, 1]} & \text { if } \theta \in[0, \pi / 2) \cup[\pi, 3 \pi / 2), \\ {[0,1-\tan \theta]} & \text { if } \theta \in(\pi / 2, \pi) \cup[3 \pi / 2,2 \pi) .\end{cases}
$$

Then for any $\theta \neq \pi / 2,3 \pi / 2$, we have $F_{\theta, a} \neq \varnothing \Longleftrightarrow a \in J_{\theta}$.

In the paper, we focus on the intersections of the Sierpinski carpet with lines of rational slope. When both $\tan \theta$ and $a$ are rational, $[6]$ proved that $F_{\theta, a}$ has a graph-directed structure $[\mathbf{1 0}]$, and the corresponding dimension is obtained.

The intersections of some special planar sets with lines in a fixed direction are studied in $[\mathbf{3}],[\mathbf{1}]$ and [5], among other publications. For example, $[\mathbf{3}]$ proved that $\operatorname{dim}_{\mathrm{H}}\left[L_{\pi / 4, a} \cap\right.$ $(C \times C)]=\log 2 /(3 \log 3)$ for almost all $a \in[-1,1]$, where $C$ is the Cantor ternary set. 

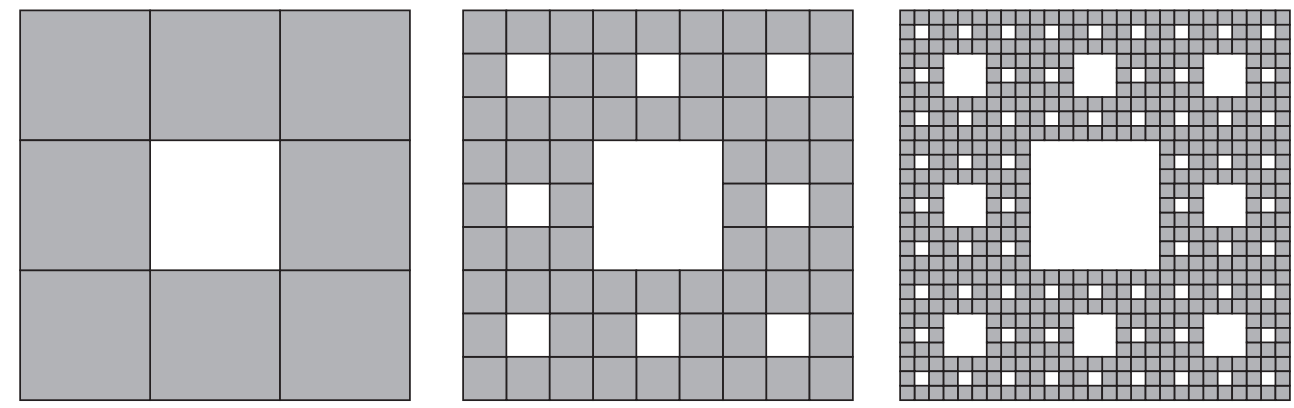

Figure 1. The steps for generating the Sierpinski carpet.

In [1] the dimensions of fibres $F_{x}=\{y \in[0,1]:(x, y) \in F\}$ for almost all $x \in[0,1]$ were discussed for some certain geometric constructions in the unit square $[0,1] \times[0,1]$. As shown in [5], we can calculate the typical value of the Hausdorff dimension of $L_{\pi / 4, a} \cap F$ for almost all $a \in J_{\pi / 4}$. In the literature listed above, $\tan \theta=0$ or 1 , but how about the general case for $\tan \theta \in \mathbb{Q}$ ? This question is the motivation for this paper.

The main result of paper is as follows.

Theorem 1.1. Suppose $F$ is the Sierpinski carpet in the plane and that $\tan \theta=$ $M / N>0$ is rational with $N, M \in \mathbb{N}$. Let $A_{0}, A_{1}$ and $A_{2}$ be $(M+N) \times(M+N)$ nonnegative integer matrices defined by $A_{t}=\left(c_{p q}^{t}\right)_{1 \leqslant p, q \leqslant N+M}$ and $c_{p q}^{t}=\#\left\{i: x_{i} M-y_{i} N=\right.$ $2 M+2+q-3 p-t\}$. Then we have the following.

(1) If

$$
a=\frac{-M-1+k}{N}+\frac{1}{N}\left(\sum_{i=1}^{\infty} x_{i} 3^{-i}\right)
$$

with $k \in \mathbb{N} \cap[1, N+M]$ and if $\left\{x_{i}\right\}_{i \geqslant 1} \in\{0,1,2\}^{\mathbb{N}}$ satisfies $3^{n} a N \notin \mathbb{Z}$ for all $n \in \mathbb{N}$, then

$$
\overline{\operatorname{dim}}_{\mathrm{B}} F_{\theta, a}=\varlimsup_{n \rightarrow \infty} \frac{\log \left\|e_{k} A_{x_{1}} A_{x_{2}} \cdots A_{x_{n}}\right\|}{n \log 3},
$$

where $e_{k}=\left(\delta_{k, 1}, \delta_{k, 2}, \ldots, \delta_{k, N+M}\right)$ is the $k$ th natural basis of $\mathbb{R}^{N+M}$.

(2) Denote by $\mathcal{L}$ the Lebesgue measure, then for $\mathcal{L}$-a.e. $a \in J_{\theta}$,

$$
\operatorname{dim}_{\mathrm{B}} F_{\theta, a}=\gamma / \log 3 \leqslant \log 8 / \log 3-1,
$$

where $\gamma$ is the Lyapunov exponent for the symmetric independent random product of $A_{0}, A_{1}, A_{2}$, i.e.

$$
\gamma=\lim _{n \rightarrow \infty} \frac{\log \left\|A_{x_{1}} A_{x_{2}} \cdots A_{x_{n}}\right\|}{n}
$$

with $x_{n}$ i.i.d. random variables assuming the values $\{0,1,2\}$ with equal probabilities.

(3) For L-a.e. $a \in J_{\theta}, \operatorname{dim}_{\mathrm{H}} F_{\theta, a}=\operatorname{dim}_{\mathrm{B}} F_{\theta, a}$. 
Remark 1.2. The results for $\theta \in(\pi / 2,2 \pi)$ are the same.

Remark 1.3. The Marstrand theorem $[\mathbf{8}, \mathbf{9}]$ concerns dimensions of sections for almost all $\theta$, where $\theta$ is random.

Remark 1.4. By using the Hutchinson metric of fractal measures, we can compute the Lyapunov exponent in special cases and we obtain

$$
\begin{array}{lll}
\text { when } \tan \theta=1, & \text { for a.e. } a \in[-1,1], & \operatorname{dim}_{\mathrm{H}} F_{\theta, a}=0.8858 \ldots, \\
\text { when } \tan \theta=\frac{1}{2}, & \text { for a.e. } a \in\left[-\frac{1}{2}, 1\right], & \operatorname{dim}_{\mathrm{H}} F_{\theta, a}=0.8914 \ldots, \\
\text { when } \tan \theta=\frac{1}{3}, & \text { for a.e. } a \in\left[-\frac{1}{3}, 1\right], & \operatorname{dim}_{\mathrm{H}} F_{\theta, a}=0.8926 \ldots ;
\end{array}
$$

all of them are less than $\log 8 / \log 3-1=0.8927 \ldots$

The paper is organized as follows. Section 2 is gives some preliminary information about the box dimension. In $\S 3$, we prove Theorem 1.1(1). In $\S 4$, Theorem 1.1(2) is proved using our key lemma: Lemma 4.2 of ergodic type. Section 5 is devoted to the proof of Theorem 1.1(3). In $\S 6$, we describe the method mentioned in Remark 1.4. In the final section, we point out that our method can apply to fractals like the Sierpinski carpet.

\section{Preliminaries}

In this section, we do not need the condition that the slope $\tan \theta$ is rational. For $i=$ $1, \ldots, 8$, let $\phi_{i}(x, y)=\frac{1}{3}(x, y)+\frac{1}{3}\left(x_{i}, y_{i}\right)$, where $\left(x_{i}, y_{i}\right) \in\{0,1,2\} \times\{0,1,2\} \backslash\{(1,1)\}$.

Fix any $\theta \in[0,2 \pi) \backslash\{\pi / 2,3 \pi / 3\}$, set $T_{i}(x)=3 x+x_{i} \tan \theta-y_{i}$, then $S_{i}=\left(T_{i}\right)^{-1}$ : $J_{\theta} \rightarrow J_{\theta}$ are linear contractions satisfying

$$
J_{\theta}=\bigcup_{i=1}^{8} S_{i}\left(J_{\theta}\right) .
$$

Let $m_{\theta}$ denote the normalized Lebesgue measure on $J_{\theta}$, i.e. $m_{\theta}=\mathcal{L} /\left|J_{\theta}\right|$ with $m_{\theta}\left(J_{\theta}\right)=1$. Write $\phi_{i_{1} \cdots i_{n}}=\phi_{i_{1}} \circ \cdots \circ \phi_{i_{n}}$.

Let $N_{n}(a)=\#\left\{i_{1} \cdots i_{n}: \phi_{i_{1} \cdots i_{n}}([0,1] \times[0,1]) \cap L_{\theta, a} \neq \varnothing\right\}$. Denote by $K_{n}(a)$ the number of 3 -adic squares of side length $3^{-n}$ intersecting $F \cap L_{\theta, a}$.

Since $\phi_{i_{1} \cdots i_{n}}([0,1] \times[0,1]) \cap L_{\theta, a} \neq \varnothing$ implies $\phi_{i_{1} \cdots i_{n}}(F) \cap L_{\theta, a} \neq \varnothing$, we have $N_{n}(a) \leqslant$ $K_{n}(a) \leqslant 9 N_{n}(a)$. It follows from the definition of the box dimension that

$$
\begin{aligned}
& \overline{\operatorname{dim}}_{\mathrm{B}}\left(F \cap L_{\theta, a}\right)=\varlimsup_{n \rightarrow \infty} \frac{\log K_{n}(a)}{n \log 3}=\varlimsup_{n \rightarrow \infty} \frac{\log N_{n}(a)}{n \log 3}, \\
& \underline{\operatorname{dim}}_{\mathrm{B}}\left(F \cap L_{\theta, a}\right)=\varliminf_{n \rightarrow \infty} \frac{\log K_{n}(a)}{n \log 3}=\varliminf_{n \rightarrow \infty} \frac{\log N_{n}(a)}{n \log 3} .
\end{aligned}
$$

We have the following lemma. 


\section{Lemma 2.1.}

$$
\begin{gathered}
N_{n}(a)=\sum_{i_{1} \cdots i_{n}} 1_{S_{i_{1} \cdots i_{n}}\left(J_{\theta}\right)}(a) . \\
\int_{J_{\theta}} N_{n}(a) \mathrm{d} m_{\theta}(a)=\left(\frac{8}{3}\right)^{n} .
\end{gathered}
$$

Proof. By induction, it is easy to verify that

$$
L_{\theta, a} \cap \phi_{i_{1} \cdots i_{n}}([0,1] \times[0,1]) \neq \varnothing \quad \Longleftrightarrow a \in S_{i_{1} \cdots i_{n}}\left(J_{\theta}\right) .
$$

Therefore,

$$
\begin{aligned}
N_{n}(a) & =\#\left\{i_{1} \cdots i_{n}: L_{\theta, a} \cap \phi_{i_{1} \cdots i_{n}}([0,1] \times[0,1]) \neq \varnothing\right\} \\
& =\#\left\{i_{1} \cdots i_{n}: a \in S_{i_{1} \cdots i_{n}}\left(J_{\theta}\right)\right\} \\
& =\sum_{i_{1} \cdots i_{n}} 1_{S_{i_{1} \cdots i_{n}}\left(J_{\theta}\right)}(a) .
\end{aligned}
$$

And thus,

$$
\int N_{n}(a) \mathrm{d} m_{\theta}(a)=\frac{1}{\left|J_{\theta}\right|} \sum_{i_{1} \cdots i_{n}} \int 1_{S_{i_{1} \cdots i_{n}}\left(J_{\theta}\right)}(a) \mathrm{d} \mathcal{L}(a)=\left(\frac{8}{3}\right)^{n} .
$$

\section{The upper box dimension}

In this section, we prove Theorem 1.1(1). Without loss of generality, we suppose that $\tan \theta=M / N>0$ is rational, where $M, N \in \mathbb{N}$ with $(M, N)=1$. Here $J_{\theta}=[-M / N, 1]$.

Let $D=\left\{a \in \mathbb{R}: 3^{n}(a N) \notin \mathbb{Z}\right.$ for any integer $\left.n \geqslant 0\right\}$.

Lemma 3.1. $\mathbb{R} \backslash D$ is an enumerable set. If $a \in D$ and $n \in\{0\} \cup \mathbb{N}$, then $3^{n} a \in D$.

For any $a \in D$, let $\Gamma_{a}=\left\{a+i / N \in J_{\theta}: i \in \mathbb{Z}\right\}$, then $\# \Gamma_{a}=(1+(M / N)) /(1 / N)=$ $(N+M)$ since $a \notin \mathbb{Z} / N$. Therefore, for any integer $n \geqslant 0$, we have $\# \Gamma_{3^{n} a}=(N+M)$ as $3^{n} a \in D$.

Given $a \in D$, we arrange the elements of $\Gamma_{a}$ as follows:

$$
\Gamma_{a}(1)<\Gamma_{a}(2)<\cdots<\Gamma_{a}(N+M),
$$

where

$$
\Gamma_{a}(i) \in\left(\frac{-M-1+i}{N}, \frac{-M+i}{N}\right) \hat{=} I_{i} \quad(1 \leqslant i \leqslant N+M)
$$


Notice that $\phi_{j}(F) \cap L_{\theta, a} \neq \varnothing \Leftrightarrow a \in S_{j}\left(J_{\theta}\right)$ and $\phi_{j}^{-1}\left(L_{\theta, a}\right)=L_{\theta, T_{j}(a)}$. Hence, for any $a \in J_{\theta}$, we have

$$
\begin{aligned}
F_{\theta, a} & =F \cap L_{\theta, a} \\
& =\left[\bigcup_{j=1}^{8} \phi_{j}(F)\right] \cap L_{\theta, a} \\
& =\bigcup_{j=1}^{8}\left[\phi_{j}(F) \cap L_{\theta, a}\right] \\
& =\bigcup_{j \text { s.t. } a \in S_{j}\left(J_{\theta}\right)} \phi_{j}\left[F \cap \phi_{j}^{-1}\left(L_{\theta, a}\right)\right] \\
& =\bigcup_{j \text { s.t. } a \in S_{j}\left(J_{\theta}\right)} \phi_{j}\left(F \cap L_{\theta, T_{j} a}\right) \\
& =\bigcup_{j \text { s.t. } a \in S_{j}\left(J_{\theta}\right)} \phi_{j}\left(F_{\theta, T_{j} a}\right) .
\end{aligned}
$$

In particular, for any $a \in J_{\theta}$, as $a=T_{i}\left(S_{i}(a)\right)$,

$$
F_{\theta, S_{i}(a)} \supset \phi_{i}\left(F_{\theta, a}\right) .
$$

If $b \in \Gamma_{a}$ and $T_{i}(b) \in J_{\theta}$, then $T_{i}(b) \in \Gamma_{3 a}$.

We know that, for any $a \in J_{\theta}, F_{\theta, a}$ is composed of some reduced (with ratio $\frac{1}{3}$ ) copies of $F_{\theta, b}$ for some $b \in \Gamma_{3 a}$. We record the number of copies with the following matrix: given $a \in J_{\theta}$, let $A(a)=\left(c_{p q}\right)_{1 \leqslant p, q \leqslant N+M}$ be a non-negative integer matrix defined by

$$
c_{p q}=\#\left\{i: T_{i}\left(\Gamma_{a}(p)\right)=\Gamma_{3 a}(q)\right\},
$$

where $c_{p q}$ is just the number of the reduced copies of $F_{\theta, \Gamma_{3 a}(q)}$ that are contained in $F_{\theta, \Gamma_{a}(p)}$, since

$$
F_{\theta, \Gamma_{a}(p)}=\bigcup_{i \text { s.t. } \Gamma_{a}(p) \in S_{i}\left(J_{\theta}\right)} \phi_{i}\left(F_{\theta, T_{i}\left(\Gamma_{a}(p)\right)}\right)
$$

and $T_{i}\left(\Gamma_{a}(p)\right)=\Gamma_{3 a}(q)$ implies $\Gamma_{a}(p) \in S_{i}\left(J_{\theta}\right)$.

Remark 3.2. We can see that for any $a \in J_{\theta}, F_{\theta, a}$ is a multi-type Moran set with generating matrices $\left\{A\left(3^{n} a\right)\right\}_{n \geqslant 0}$ and constant ratio $\frac{1}{3}$. Please refer to $[\mathbf{7}]$ for the definition of multi-type Moran sets. If $\left\{A\left(3^{n} a\right)\right\}_{n} \geqslant 0$ is an ultimately periodic sequence, e.g. when $a \in D$ is rational as in $[6]$, then $F_{\theta, a}$ can be characterized as a graph-directed set.

For any $a \in D \cap J_{\theta}$, let $i_{0}(a)$ be the integer satisfying

$$
\Gamma_{a}\left(i_{0}(a)\right)=a .
$$

Then

$$
N_{n}(a)=\left\|e_{i_{0}(a)} A(a) A(3 a) \cdots A\left(3^{n-1} a\right)\right\|_{1},
$$


where the norm of the row vector is given by

$$
\left\|\left(x_{1}, \ldots, x_{N+M}\right)\right\|_{1}=\sum_{i}\left|x_{i}\right| .
$$

For any $x \in \mathbb{R}$, let $x(\bmod (1 / N))$ denote the unique value $x^{\prime} \in[0,1 / N)$ with $x \equiv$ $x^{\prime} \bmod (1 / N)$. Here $x \equiv y \bmod (1 / N)$ means that $N(x-y)$ is an integer.

Given any $a \in D \cap J_{\theta}$, we write

$$
a=\frac{-M-1+k}{N}+\frac{1}{N}\left(\sum_{i=1}^{\infty} x_{i} 3^{-i}\right)
$$

with $k \in \mathbb{N} \cap[1, N+M]$ and $\left\{x_{i}\right\}_{i \geqslant 1} \in\{0,1,2\}^{\mathbb{N}}$.

We have the following properties.

(1) Suppose $x, y \in D$, then

$$
x \equiv y \bmod (1 / N) \Longrightarrow \Gamma_{x}=\Gamma_{y} \text { and } A(x)=A(y) .
$$

Furthermore, given $j \in\{0,1,2\}$,

$$
A(x) \text { is constant on }\left\{x \in D: x(\bmod (1 / N)) \in\left(\frac{j}{3 N}, \frac{j+1}{3 N}\right)\right\},
$$

since, for any $\left(x_{i}, y_{i}\right) \in\{0,1,2\} \times\{0,1,2\} \backslash\{(1,1)\}$ and $\eta \in \mathbb{Z}$,

$$
3\left(\frac{\eta}{3 N}, \frac{\eta+1}{3 N}\right)+x_{i} \frac{M}{N}-y_{i}=\left(\frac{k}{N}, \frac{k-1}{N}\right) \text { for some } k \in \mathbb{Z} .
$$

Denote by $A_{j}$ the above constant matrix. In fact, define intervals

$I_{q}=\left(\frac{-M-1+q}{N}, \frac{-M+q}{N}\right)$ and $J_{p}^{t}=\left(\frac{-M-1+p}{N}+\frac{t}{3 N}, \frac{-M-1+p}{N}+\frac{t+1}{3 N}\right)$

for $p, q \in \mathbb{N} \cap[1, N+M]$ and $t \in\{0,1,2\}$. Then $A_{t}=\left(c_{p q}^{t}\right)_{1 \leqslant p, q \leqslant N+M}$ with

$$
c_{p q}^{t}=\#\left\{i: T_{i}\left(J_{p}^{t}\right)=I_{q}\right\},
$$

i.e. $c_{p q}^{t}=\#\left\{i: x_{i} M-y_{i} N=2 M+2+q-3 p-t\right\}$. That means that, for each $j$, the matrix $A_{j}$ is the same one in Theorem 1.1.

Hence, for

$$
b=\frac{M^{\prime}}{N}+\frac{1}{N}\left(\sum_{i=1}^{\infty} x_{i} 3^{-i}\right) \in D
$$

with $M^{\prime} \in \mathbb{Z}$ and $\left\{x_{i}\right\}_{i} \in\{0,1,2\}^{\mathbb{N}}$, we have

$$
A(b)=A_{x_{1}} .
$$

(2) Let $\mu=\left.N \cdot \mathcal{L}\right|_{[0,1 / N)}$ and let $T(x)=3 x \bmod (1 / N)$. Then

$$
(\mathbb{R} / \bmod (1 / N), T, \mu) \text { is ergodic, }
$$

since $(\mathbb{R} / \bmod (1 / N), T, \mu) \simeq(\mathbb{R} / \bmod (1), x \rightarrow 3 x(\bmod 1), \mathcal{L})$. 
(3) For $x \in D / \bmod (1 / N)$, let

$$
A_{n}(x)=A(x) A(3 x) \cdots A\left(3^{n-1} x\right)=A(x) A(T x) \cdots A\left(T^{n-1} x\right) .
$$

Then $\left\{A_{n}(\cdot)\right\}_{n}$ are measurable functions defined on $D / \bmod (1 / N)$, a subset of full measure contained in $(\mathbb{R} / \bmod (1 / N), \mu)$.

We will prove Theorem 1.1(1). For

$$
a=\frac{-M-1+k}{N}+\frac{1}{N}\left(\sum_{i=1}^{\infty} x_{i} 3^{-i}\right)
$$

it follows from (2.1), (3.1) and (3.3) that

$$
\begin{aligned}
\overline{\operatorname{dim}}_{\mathrm{B}} F_{\theta, a} & =\varlimsup_{n \rightarrow \infty} \frac{\log N_{n}(a)}{n \log 3} \\
& =\varlimsup_{n \rightarrow \infty} \frac{\log \left\|e_{i_{0}(a)} A(a) A(3 a) \cdots A\left(3^{n-1} a\right)\right\|_{1}}{n \log 3} \\
& =\varlimsup_{n \rightarrow \infty} \frac{\log \left\|e_{k} A_{x_{1}} A_{x_{2}} \cdots A_{x_{n}}\right\|_{1}}{n \log 3},
\end{aligned}
$$

where $i_{0}(a)=k$ and $A\left(3^{j} a\right)=A_{x_{j+1}}$ for any $j \in\{0\} \cup \mathbb{N}$.

Replacing $\|\cdot\|_{1}$ by any norm $\|\cdot\|$ of $\mathbb{R}^{N+M}$, we get

$$
\overline{\operatorname{dim}}_{\mathrm{B}} F_{\theta, a}=\varlimsup_{n \rightarrow \infty} \frac{\log \left\|e_{k} A_{x_{1}} A_{x_{2}} \cdots A_{x_{n}}\right\|}{n \log 3} .
$$

This completes the proof of Theorem $1.1(1)$.

\section{A typical value of the box dimension}

For any real matrix $A_{k \times k}=\left(a_{i j}\right)_{1 \leqslant i, j \leqslant k}$, let $\|A\|=\sum_{i j}\left|a_{i j}\right|$. Then for any matrices $A_{k \times k}, B_{k \times k}$, we have $\|A B\| \leqslant\|A\| \cdot\|B\|$.

Lemma 4.1. For $\mathcal{L}$ almost all $a \in J_{\theta}$,

$$
\varlimsup_{n \rightarrow \infty} \frac{\log N_{n}(a)}{n} \leqslant \lim _{n \rightarrow \infty} \frac{\log \left\|A_{n}(a)\right\|}{n}=\gamma \leqslant \log \left(\frac{8}{3}\right) .
$$

Here $\gamma$ is the Lyapunov exponent with respect to $A_{0}, A_{1}$ and $A_{2}$.

Proof. Now $A_{n}(\cdot)$ is defined on $D / \bmod (1 / N)$, a set of full measure contained in $(\mathbb{R} / \bmod (1 / N), \mu)$. Moreover,

$$
A_{n+m}(a)=A_{n}(a) A_{m}\left(3^{n} a\right)=A_{n}(a) A_{m}\left(T^{n} a\right)
$$

for $a \in D / \bmod (1 / N)$. Therefore, we have

$$
\log \left\|A_{n+m}(a)\right\| \leqslant \log \left\|A_{n}(a)\right\|+\log \left\|A_{m}\left(T^{n} a\right)\right\| .
$$


It follows from the subadditive ergodic theorem [11] that there exists a constant $\gamma$ such that

$$
\gamma=\lim _{n \rightarrow \infty} \frac{\log \left\|A_{n}(a)\right\|}{n}=\lim _{n \rightarrow \infty} \frac{1}{n} \int \log \left\|A_{n}(a)\right\| \mathrm{d} \mu(a)
$$

for $\mu$ almost all $a \in D / \bmod (1 / N)$. This means that $\gamma$ is the corresponding Lyapunov exponent with respect to $A_{0}, A_{1}$ and $A_{2}$, which is independent of the given matrix norm.

By the definition of $\mu$ and (3.2), for $\mathcal{L}$ almost all $a \in J_{\theta}$,

$$
\gamma=\lim _{n \rightarrow \infty} \frac{\log \left\|A_{n}(a)\right\|}{n}=\lim _{n \rightarrow \infty} \frac{1}{n} \int_{J_{\theta}} \log \left\|A_{n}(a)\right\| \mathrm{d} m_{\theta}(a) .
$$

By the definition of $A_{n}(a)$,

$$
\left\|A_{n}(a)\right\|=\sum_{b \in \Gamma_{a}} N_{n}(b) .
$$

As $\log (x)$ is convex, $\int \log f(x) \leqslant \log \int f(x)$. By using this inequality, (2.4), (4.1) and (4.2), we have

$$
\begin{aligned}
\gamma & =\lim _{n \rightarrow \infty} \frac{1}{n} \int_{J_{\theta}} \log \left\|A_{n}(a)\right\| \mathrm{d} m_{\theta}(a) \\
& \leqslant \lim _{n \rightarrow \infty} \frac{1}{n} \log \left[\int_{J_{\theta}}\left\|A_{n}(a)\right\| \mathrm{d} m_{\theta}(a)\right] \\
& =\lim _{n \rightarrow \infty} \frac{1}{n} \log \left[\int_{J_{\theta}} \sum_{b \in \Gamma_{a}} N_{n}(b) \mathrm{d} m_{\theta}(a)\right] \\
& =\lim _{n \rightarrow \infty} \frac{1}{n} \log \left[(N+M)\left(\frac{8}{3}\right)^{n}\right] \\
& =\log \left(\frac{8}{3}\right) .
\end{aligned}
$$

In addition, for $\mathcal{L}$ almost all $a \in J$,

$$
\varlimsup_{n \rightarrow \infty} \frac{\log N_{n}(a)}{n} \leqslant \lim _{n \rightarrow \infty} \frac{\log \sum_{b \in \Gamma_{a}} N_{n}(b)}{n}=\lim _{n \rightarrow \infty} \frac{\log \left\|A_{n}(a)\right\|}{n}=\gamma .
$$

Lemma 4.2. Suppose that $B \subset J_{\theta}$ is a $\mathcal{L}$-measurable set with $m_{\theta}(B)>0$. If $\bigcup_{i} S_{i}(B) \subset B$, then $m_{\theta}(B)=1$.

Proof. Suppose that $m_{\theta}(B)<1$, then $0<m_{\theta}\left(B^{\mathrm{c}}\right)<1$ and $\mathcal{L}\left(J_{\theta} \cap B^{\mathrm{c}}\right)>0$. As $\bigcup_{i} S_{i}(B) \subset B$, we have

$$
S_{i_{1} \cdots i_{k}}^{-1}\left(B^{\mathrm{c}}\right) \subset B^{\mathrm{c}} \quad \text { for any } i_{1} \cdots i_{k} .
$$

Since $\mathcal{L}\left(J_{\theta} \cap B^{\mathrm{c}}\right)>0$, we can take a Lebesgue point $x_{0} \in J_{\theta} \cap B^{\mathrm{c}}$ of density 1 , which implies that for any $\delta>0$ there exists $\varepsilon_{0}>0$ such that

$$
\mathcal{L}\left[I \cap\left(J_{\theta} \cap B^{\mathrm{c}}\right)\right] /|I| \geqslant 1-\delta
$$

whenever $x_{0} \in I$ with $|I| \leqslant \varepsilon_{0}$. 
Given a sufficiently large integer $k$, as $J_{\theta}=\bigcup_{i_{1} \cdots i_{k}} S_{i_{1} \cdots i_{k}}\left(J_{\theta}\right)$, we can select an interval $I=S_{j_{1} \cdots j_{k}}\left(J_{\theta}\right) \subset J_{\theta}$ such that $x_{0} \in I$ with

$$
|I|=3^{-k}\left|J_{\theta}\right|<\varepsilon_{0},
$$

then, by (4.4),

$$
\mathcal{L}\left(I \cap B^{\mathrm{c}}\right) /|I|=\mathcal{L}\left(I \cap J_{\theta} \cap B^{\mathrm{c}}\right) /|I| \geqslant 1-\delta .
$$

By using (4.3), we have

$$
\left(S_{j_{1} \cdots j_{k}}\right)^{-1}\left(I \cap B^{\mathrm{c}}\right)=\left(S_{j_{1} \cdots j_{k}}\right)^{-1}\left(S_{j_{1} \cdots j_{k}}\left(J_{\theta}\right) \cap B^{\mathrm{c}}\right) \subset J_{\theta} \cap B^{\mathrm{c}} .
$$

Hence,

$$
\frac{\mathcal{L}\left(J_{\theta} \cap B^{\mathrm{c}}\right)}{\left|J_{\theta}\right|} \geqslant \frac{\mathcal{L}\left[\left(S_{j_{1} \cdots j_{k}}\right)^{-1}\left(I \cap B^{\mathrm{c}}\right)\right]}{\mathcal{L}\left[\left(S_{j_{1} \cdots j_{k}}\right)^{-1}(I)\right]}=\frac{3^{n} \mathcal{L}\left(I \cap B^{\mathrm{c}}\right)}{3^{n}|I|} \geqslant(1-\delta) .
$$

This implies that

$$
m_{\theta}\left(B^{\mathrm{c}}\right) \geqslant 1-\delta .
$$

Letting $\delta \rightarrow 0$, we have $m_{\theta}\left(B^{\mathrm{c}}\right)=1$. This yields a contradiction.

Proposition 4.3. For $\mathcal{L}$ almost all $a \in J_{\theta}$,

$$
\overline{\operatorname{dim}}_{\mathrm{B}} F_{\theta, a}=\gamma / \log 3,
$$

where $\gamma$ is the Lyapunov exponent with respect to $A_{0}, A_{1}$ and $A_{2}$.

Proof. By (2.1) and Lemma 4.1, $\overline{\operatorname{dim}}_{\mathrm{B}} F_{\theta, a} \leqslant \gamma / \log 3$ for $\mathcal{L}$ almost all $a$. So we need to prove only that $\overline{\operatorname{dim}}_{\mathrm{B}} F_{\theta, a} \geqslant \gamma / \log 3$ for $\mathcal{L}$ almost all $a$. By (4.2) and Lemma 4.1, for $\mathcal{L}$ almost all $a \in J_{\theta}$, we have

$$
\max _{b \in \Gamma_{a}} \overline{\operatorname{dim}}_{\mathrm{B}} F_{\theta, b}=\varlimsup_{n \rightarrow \infty} \frac{\log \left\|A_{n}(a)\right\|}{n \log 3}=\frac{\gamma}{\log 3} .
$$

Let

$$
B=\left\{a \in J_{\theta}: \overline{\operatorname{dim}}_{\mathrm{B}} F_{\theta, a} \geqslant \gamma / \log 3\right\},
$$

which is an $\mathcal{L}$-measurable set. It follows from (4.5) that there is a set $K$ of zero Lebesgue measure such that

$$
J_{\theta} \backslash K \subset \bigcup_{i=-(N+M)}^{N+M}\left(B+\frac{i}{N}\right)
$$

where $B+x=\{b+x: b \in B\}$. Hence

$$
0<\mathcal{L}\left(J_{\theta}\right) \leqslant \sum_{i=-(N+M)}^{N+M} \mathcal{L}\left(B+\frac{i}{N}\right)=(2 N+2 M+1) \mathcal{L}(B),
$$

which implies that

$$
m_{\theta}(B)=\mathcal{L}(B) /\left|J_{\theta}\right|>0
$$


By using $(*)$ in $\S 3$, we have $\overline{\operatorname{dim}}_{\mathrm{B}} F_{\theta, S_{i}(a)} \geqslant \overline{\operatorname{dim}}_{\mathrm{B}} F_{\theta, a}$, i.e.

$$
\bigcup_{i} S_{i}(B) \subset B .
$$

It follows from Lemma 4.2 that $m_{\theta}(B)=1$.

Proposition 4.4. For $\mathcal{L}$ almost all $a \in[-\tan \theta, 1]$,

$$
\operatorname{dim}_{\mathrm{B}} F_{\theta, a}=\gamma / \log 3 .
$$

Proof. Notice that $A_{n+m}(a)=A_{n}(a) A_{m}\left(T^{n} a\right)$, and $(\mathbb{R} / \bmod (1 / N), T, \mu)$ is ergodic. Then it follows from the multiplicative ergodic theorem [11] that for each $e_{i}(1 \leqslant i \leqslant$ $N+M$ ), and for $\mu$-almost all (i.e. $\mathcal{L}$-almost all) $a \in[0,1 / N) \cap D$,

$$
\lim _{n \rightarrow \infty} \frac{\log \left\|e_{i} A_{n}(a)\right\|}{n}=\lambda(a, i),
$$

where $\lambda(a, i)$ depends on $a$ and $e_{i}$. It follows from (2.1), (2.2), (3.1), (4.6) and Proposition 4.3 that, for $\mathcal{L}$ almost all $a$,

$$
\operatorname{dim}_{\mathrm{B}} F_{\theta, a}=\gamma / \log 3 .
$$

\section{Equality of the Hausdorff and box dimensions}

In this section we will prove Theorem $1.1(3)$, i.e. for a fixed rational slope $\tan \theta$ and almost all $a \in J_{\theta}$, the Hausdorff dimension and the upper box dimension of the section $F_{\theta, a}$ are equal. To prove this, we shall use Proposition 2.6 in [5] provided by Ledrappier.

Let $T_{3}$ denote the endomorphism $T_{3} x=3 x(\bmod 1)$ of the one-dimensional torus $\mathbb{T}=$ $\mathbb{R} /(\bmod 1)$, and let $S$ be a continuous transformation of a metric space $Y$. Assume that $\Lambda \subset \mathbb{T} \times Y$ is compact and invariant under the map $T_{3} \times S$, and that $\nu$ is an $S$-invariant probability measure on $Y$. Then for $\nu$-a.e. $y$, we have

$$
\operatorname{dim}_{\mathrm{H}}\left[\pi^{-1}(y)\right]=\operatorname{dim}_{\mathrm{B}}\left[\pi^{-1}(y)\right],
$$

where $\pi: \Lambda \rightarrow Y$ is the projection onto the second coordinate.

Proof of Theorem 1.1 (3). At first, we will show that, for almost all $a \in[-M / N, 1]$,

$$
\max _{b \in \Gamma_{a}} \operatorname{dim}_{\mathrm{B}} F_{\theta, b}=\max _{b \in \Gamma_{a}} \operatorname{dim}_{\mathrm{H}} F_{\theta, b} .
$$

In fact, fix $a \in[-M / N, 1]$, we have

$$
\begin{aligned}
\bigcup_{b \in \Gamma_{a}} F_{\theta, b} & =\bigcup_{i \in \mathbb{Z}}[F \cap\{(x, y): y=(M / N) x+i / N+a\}] \\
& =F \cap\{(x, y): N y-M x \equiv a N(\bmod 1)\} .
\end{aligned}
$$


Let $T_{3}(x)=3 x(\bmod 1)$ be a map on the one-dimensional torus $\mathbb{T}$, and let $T=T_{3} \times T_{3}$ and $P=(x,(N y-M x)(\bmod 1))$ be the maps on the two-dimensional torus $\mathbb{T}^{2}$. Then the Sierpinski carpet $F$ and its image $P(F)$ are invariant under $T$. Here $P$ is a bi-Lipschitz endomorphism when restricted to a subset

$$
\left\{(x, y) \in \mathbb{T}^{2}: y \in[j / N,(j+1) / N)\right\}
$$

for each integer $j \in[0, N-1]$. Therefore, let 'dim' stand for any one of $\operatorname{dim}_{\mathrm{H}}$, $\overline{\operatorname{dim}}_{\mathrm{B}}$ and $\underline{\operatorname{dim}}_{B}$,

$$
\operatorname{dim} P\left(\bigcup_{b \in \Gamma_{a}} F_{\theta, b}\right)=\operatorname{dim}\left(\bigcup_{b \in \Gamma_{a}} F_{\theta, b}\right) .
$$

We now prepare to apply the stated result from [5]. Let $S=T_{3}$ on $Y=\mathbb{T}$ equipped with a normalized Lebesgue measure $\nu$, then $T=T_{3} \times T_{3}=T_{3} \times S, P(F) \subset \mathbb{T} \times Y=\mathbb{T}^{2}$ is compact and invariant under $T$, and

$$
\pi^{-1}[N a(\bmod 1)]=P\left(\bigcup_{b \in \Gamma_{a}} F_{\theta, b}\right) .
$$

Applying the result from [5], we have, for almost all $a$,

$$
\operatorname{dim}_{\mathrm{H}} P\left(\bigcup_{b \in \Gamma_{a}} F_{\theta, b}\right)=\operatorname{dim}_{\mathrm{B}} P\left(\bigcup_{b \in \Gamma_{a}} F_{\theta, b}\right) .
$$

Since the low box dimension lies between the Hausdorff and upper box dimensions, by using (5.2) and (5.3), we obtain (5.1). Hence, for $\mathcal{L}$ almost all $a \in J_{\theta}$,

$$
\max _{b \in \Gamma_{a}} \operatorname{dim}_{\mathrm{H}} F_{\theta, b}=\operatorname{dim}_{\mathrm{B}} F_{\theta, a}=\gamma / \log 3 .
$$

By an argument analogous to Proposition 4.3, we have

$$
\operatorname{dim}_{\mathrm{H}} F_{\theta, a}=\operatorname{dim}_{\mathrm{B}} F_{\theta, a}=\gamma / \log 3 \quad \text { for } \mathcal{L} \text { almost all } a \in J_{\theta} .
$$

\section{Computation of the Lyapunov exponent and dimension}

In this section, we give a method mentioned in Remark 1.4 following Theorem 1.1. Suppose $\tan \theta=N / M>0$. Let $n=N+M$. Then $\left\{A_{i}\right\}_{i=0}^{2}$ are non-negative integer $n \times n$ matrices.

For $x=\left(x_{1}, \ldots, x_{n}\right)^{\mathrm{T}}$, let $\|x\|_{1}=\sum_{i}\left|x_{i}\right|$. We define $\Delta_{n-1}=\left\{\left(x_{1}, \ldots, x_{n}\right)^{\mathrm{T}} \mid x_{i} \geqslant\right.$ 0 , for all $i$ and $\left.\|x\|_{1}=1\right\}$. Let $\mathcal{L}$ denote the Lebesgue measure on $[0,1]$. For almost all $t \in[0,1]$, write $t=\sum_{i=1}^{\infty} t_{i} 3^{-i}$ with $\left\{t_{i}\right\}_{i=1}^{\infty} \in\{0,1,2\}^{\mathbb{N}}$.

Lemma 6.1. For $i \in\{0,1,2\},\left\|A_{i} x\right\|_{1} \geqslant 1$ for all $x \in \Delta_{n-1}$. 
Proof. Given any $x=\left(x_{1}, \ldots, x_{n}\right)^{\mathrm{T}} \in \Delta_{n-1}$,

$$
\begin{aligned}
\left\|A_{i} x\right\|_{1} & =\sum_{i}\left\|A_{i}\left(0, \ldots, x_{i}, \ldots, 0\right)^{\mathrm{T}}\right\|_{1} \\
& =\sum_{i} x_{i}\left\|A_{i}(0, \ldots, 1, \ldots, 0)^{\mathrm{T}}\right\|_{1},
\end{aligned}
$$

we need only to show that $\left\|A_{i}(0, \ldots, 1, \ldots, 0)^{\mathrm{T}}\right\|_{1} \geqslant 1$.

It suffices to prove that, for any $c \in J \cap D$, every column vector of $A(c)$ is non-zero, i.e. for any $b \in \Gamma_{3 c}$ there exists some $i$ such that

$$
S_{i}(b) \in \Gamma_{c} .
$$

Suppose that $b=3 c+(i / N)$. Notice that $\left\{S_{i}: J_{\theta} \rightarrow J_{\theta}\right\}_{i}$ are contractions satisfying

$$
S_{i}(x)=\frac{1}{3} x+\frac{1}{3} y_{i}-\frac{1}{3} x_{i} \tan \theta=\frac{1}{3} x+\frac{1}{3} y_{i}-\frac{M}{3 N} x_{i}
$$

with $\left(x_{i}, y_{i}\right) \in\{0,1,2\} \times\{0,1,2\} \backslash\{(1,1)\}$.

We will distinguish three cases.

(1) When $M \equiv 1$ or $M \equiv 2(\bmod 3)$, we can always select $k \in\{0,1,2\}$ such that

$$
i-k M \equiv 0(\bmod 3) \text {. }
$$

By using the self-mapping $x \rightarrow\left(\frac{1}{3}\right) x-k(M / 3 N)$ on $J_{\theta}$, we have

$$
\left(\frac{1}{3}\right) b-k \frac{M}{3 N}=c+\frac{(i-k M) / 3}{N} \in \Gamma_{c} .
$$

(2) When $N \equiv 1$ or $N \equiv 2(\bmod 3)$, we can always select $k \in\{0,1,2\}$ such that

$$
i+k N \equiv 0(\bmod 3) .
$$

By using the self-mapping $x \rightarrow\left(\frac{1}{3}\right) x+k \frac{1}{3}$ on $J_{\theta}$, we have

$$
\left(\frac{1}{3}\right) b+k \frac{1}{3}=c+\frac{(i+k N) / 3}{N} \in \Gamma_{c} .
$$

(3) If $M, N \equiv 0(\bmod 3)$, then $1=(M, N) \geqslant 3$. This yields a contradiction.

By Lemma 6.1 , given $i \in\{0,1,2\}$, a mapping $\bar{A}_{i}: \Delta_{n-1} \rightarrow \Delta_{n-1}$ is defined by

$$
\bar{A}_{i}(x)=\frac{A_{i} x}{\left\|A_{i} x\right\|_{1}} \in \Delta_{n-1} \quad \text { for any } x \in \Delta_{n-1} .
$$

As shown in [5, p. 615], a probability measure $\nu$ on $\Delta_{n-1}$ is called a stationary measure for the random product if

$$
\nu=\int_{[0,1]} \bar{A}_{t_{1}} \nu \mathrm{d} \mathcal{L}(t)
$$


where $t_{1}$ is the first term of the 3 -adic expansion of $t$. By using the notion in [4], the above formula is

$$
\nu=\frac{1}{3}\left[\nu \circ\left(\bar{A}_{0}\right)^{-1}+\nu \circ\left(\bar{A}_{1}\right)^{-1}+\nu \circ\left(\bar{A}_{2}\right)^{-1}\right] .
$$

If the stationary measure is unique, the results of $[2]$ show that

$$
\gamma=\frac{1}{3} \int_{\Delta_{n-1}}\left(\log \left\|A_{0} x\right\|_{1}+\log \left\|A_{1} x\right\|_{1}+\log \left\|A_{2} x\right\|_{1}\right) \mathrm{d} \nu(x) .
$$

\subsection{Hutchinson's metric}

Hutchinson's metric is a kind of metric for measures [4]. Let $\delta$ be a metric on $\Delta_{n-1}$ such that $\left(\Delta_{n-1}, \delta\right)$ is a compact space. Then the diameter $\operatorname{diam}_{\delta}\left(\Delta_{n-1}\right)$ of $\Delta_{n-1}$ is finite. For any Borel probability measure $\nu_{1}, \nu_{2}$ on $\left(\Delta_{n-1}, \delta\right)$, Hutchinson's metric is defined by

$$
d_{\mathrm{H}}\left(\nu_{1}, \nu_{2}\right)=\sup _{\operatorname{Lip}(f) \leqslant 1}\left|\int f \mathrm{~d} \nu_{1}-\int f \mathrm{~d} \nu_{2}\right|,
$$

where

$$
\operatorname{Lip}(f)=\sup _{x \neq y} \frac{|f(x)-f(y)|}{\delta(x, y)}
$$

for any function $f: \Delta_{n-1} \rightarrow \mathbb{R}$.

Notice that, for any $\nu_{1}, \nu_{2}$ on $\left(\Delta_{n-1}, \delta\right)$,

$$
d_{\mathrm{H}}\left(\nu_{1}, \nu_{2}\right) \leqslant 2 \sup _{x, y \in \Delta_{n-1}}(\delta(x, y))=2 \operatorname{diam}_{\delta}\left(\Delta_{n-1}\right) .
$$

Let $\mathcal{M}^{1}$ be the collection of Borel probability measures on $\left(\Delta_{n-1}, \delta\right)$. Then the metric space $\left(\mathcal{M}^{1}, d_{\mathrm{H}}\right)$ is compact. Define $\mathcal{F}: \mathcal{M}^{1} \rightarrow \mathcal{M}^{1}$ by

$$
\mathcal{F} \nu=\frac{1}{3} \sum_{i=0}^{2} \nu \circ\left(\bar{A}_{i}\right)^{-1} .
$$

We assume that

(1) there is a constant $\tau$ such that

$$
\|x-y\|_{1} \leqslant \tau \cdot \delta(x, y) \quad \text { for all } x, y \in \Delta_{n-1}
$$

(2) $\mathcal{F}$ is contractive, i.e. there is a constant $c \in(0,1)$ such that, for any $\nu_{1}, \nu_{2} \in \mathcal{M}^{1}$,

$$
d_{\mathrm{H}}\left(\mathcal{F} \nu_{1}, \mathcal{F} \nu_{2}\right) \leqslant c d_{\mathrm{H}}\left(\nu_{1}, \nu_{2}\right)
$$

So, by $[4]$ there is a unique stationary measure, denoted by $\nu$.

Here, for any $i$,

$$
\log \left\|A_{i} x\right\|_{1}:\left(\Delta_{n-1}, \delta\right) \rightarrow \mathbb{R} \text { is Lipschitz }
$$


due to Lemma 6.1 and (6.7). In fact, suppose that $A_{i}=\left(a_{p q}\right)_{1 \leqslant p, q \leqslant n}$ and let $\left\|A_{i}\right\|^{*}=$ $\max _{q}\left(\sum_{p} a_{p q}\right)$, then, by Lemma 6.1 , for any $x, y \in \Delta_{n-1}$, we have

$$
\begin{aligned}
\left|\log \left\|A_{i} x\right\|_{1}-\log \left\|A_{i} y\right\|_{1}\right| & \leqslant\left|\left\|A_{i} x\right\|_{1}-\left\|A_{i} y\right\|_{1}\right| \\
& \leqslant \max _{q}\left(\sum_{p} a_{p q}\right)\|x-y\|_{1} \\
& \leqslant\left(\tau\left\|A_{i}\right\|^{*}\right) \cdot \delta(x, y) .
\end{aligned}
$$

Under the assumption above, we can estimate the Lyapunov exponent in the following way.

Let $\nu_{0}$ be an atom measure supported on any point of $\Delta_{n-1}$ and let $\nu_{1}=\mathcal{F} \nu_{0}, \ldots$, $\nu_{k+1}=\mathcal{F} \nu_{k}$ by induction, then

$$
\begin{aligned}
d_{\mathrm{H}}\left(\nu, \nu_{k}\right) & =d_{\mathrm{H}}\left(\mathcal{F} \nu, \mathcal{F} \nu_{k-1}\right) \\
& \leqslant c d_{\mathrm{H}}\left(\nu, \nu_{k-1}\right) \\
& \vdots \\
& \leqslant c^{k} d_{\mathrm{H}}\left(\nu, \nu_{0}\right) \leqslant 2 c^{k} \operatorname{diam}_{\delta}\left(\Delta_{n-1}\right) .
\end{aligned}
$$

Let $f(x)=\frac{1}{3} \sum_{i} \log \left\|x A_{i}\right\|_{1}$ be a function on $\Delta_{n-1}$, and let

$$
\gamma_{k}=\int f(x) \mathrm{d} \nu_{k}(x)
$$

Therefore, we have the following estimate:

$$
\begin{aligned}
\left|\gamma-\gamma_{k}\right| & =\left|\int f \mathrm{~d} \nu-\int f \mathrm{~d} \nu_{k}\right| \\
& \leqslant \operatorname{Lip}(f) d_{\mathrm{H}}\left(\nu, \nu_{k}\right) \leqslant c^{k}\left[2 \tau\left(\max _{0 \leqslant i \leqslant 2}\left\|A_{i}\right\|^{*}\right) \operatorname{diam}_{\delta}\left(\Delta_{n-1}\right)\right] .
\end{aligned}
$$

That is,

$$
\left|\gamma-\gamma_{k}\right| \leqslant c^{k}\left[2 \tau\left(\max _{0 \leqslant i \leqslant 2}\left\|A_{i}\right\|^{*}\right) \operatorname{diam}_{\delta}\left(\Delta_{n-1}\right)\right]
$$

\subsection{Example}

We mainly compute the Lyapunov exponent in the case of $\tan \theta=\frac{1}{2}$. We equip $\Delta_{2}$ with a metric defined by

$$
\delta(x, y)=\max \left\{\left|x_{1}-y_{1}\right|,\left|x_{3}-y_{3}\right|\right\}
$$

for any $x=\left(x_{1}, x_{2}, x_{3}\right)^{\mathrm{T}}, y=\left(y_{1}, y_{2}, y_{3}\right)^{\mathrm{T}} \in \Delta_{2}$. Here $\delta$ is a metric satisfying $\delta(x, y) \leqslant$ $\|x-y\|_{1} \leqslant 4 \delta(x, y)$ and $\operatorname{diam}_{\delta}\left(\Delta_{2}\right)=1$. We note that $\left(\Delta_{2}, \delta\right)$ is compact.

In the case of $\tan \theta=\frac{1}{2}$, we have

$$
A_{0}=\left[\begin{array}{lll}
1 & 0 & 0 \\
0 & 2 & 1 \\
1 & 1 & 2
\end{array}\right], \quad A_{1}=\left[\begin{array}{lll}
1 & 1 & 0 \\
2 & 0 & 2 \\
0 & 1 & 1
\end{array}\right], \quad A_{2}=\left[\begin{array}{lll}
2 & 1 & 1 \\
1 & 2 & 0 \\
0 & 0 & 1
\end{array}\right]
$$


with $\max _{0 \leqslant i \leqslant 2}\left\|A_{i}\right\|^{*}=3$. Take any $x=\left(x_{1}, x_{2}, x_{3}\right)^{\mathrm{T}}, y=\left(y_{1}, y_{2}, y_{3}\right)^{\mathrm{T}} \in \Delta_{2}$; we then obtain

$$
\begin{aligned}
& \delta\left(\bar{A}_{0}(x), \bar{A}_{0}(y)\right)=\max \left\{\left|\frac{x_{1}}{3-x_{1}}-\frac{y_{1}}{3-y_{1}}\right|,\left|\frac{1+x_{3}}{3-x_{1}}-\frac{1+y_{3}}{3-y_{1}}\right|\right\}, \\
& \delta\left(\bar{A}_{1}(x), \bar{A}_{1}(x)\right)=\max \left\{\left|\frac{1-x_{3}}{3-x_{2}}-\frac{1-y_{3}}{3-y_{2}}\right|,\left|\frac{1-x_{1}}{3-x_{2}}-\frac{1-y_{1}}{3-y_{2}}\right|\right\}, \\
& \delta\left(\bar{A}_{2}(x), \bar{A}_{2}(x)\right)=\max \left\{\left|\frac{1+x_{1}}{3-x_{3}}-\frac{1+y_{1}}{3-y_{3}}\right|,\left|\frac{x_{3}}{3-x_{3}}-\frac{y_{3}}{3-y_{3}}\right|\right\} .
\end{aligned}
$$

We have

$$
\begin{aligned}
\left|\frac{x_{1}}{3-x_{1}}-\frac{y_{1}}{3-y_{1}}\right| & =\frac{3\left(x_{1}-y_{1}\right)}{\left(3-x_{1}\right)\left(3-y_{1}\right)} \leqslant \frac{3}{4}\left|x_{1}-y_{1}\right|, \\
\left|\frac{1+x_{3}}{3-x_{1}}-\frac{1+y_{3}}{3-y_{1}}\right| & =\left|\frac{\left(1+y_{3}\right)\left(x_{1}-y_{1}\right)+\left(3-y_{1}\right)\left(x_{3}-y_{3}\right)}{\left(3-x_{1}\right)\left(3-y_{1}\right)}\right| \\
& \leqslant \frac{1+y_{3}}{\left(3-x_{1}\right)\left(3-y_{1}\right)}\left|x_{1}-y_{1}\right|+\frac{1}{3-x_{1}}\left|x_{3}-y_{3}\right| \\
\left|\frac{1-x_{3}}{3-x_{2}}-\frac{1-y_{3}}{3-y_{2}}\right| & \left.=\mid \frac{1+y_{3}}{3-y_{1}} \leqslant \frac{2-y_{1}}{3-y_{1}} \leqslant \frac{2}{3}\right) \\
& \left.\leqslant \frac{3}{4} \mid x_{3}-y_{1}\right)\left(y_{3}\left|+\frac{1}{2}\right| x_{3}-y_{3}\right)+\left(1-y_{3} \mid x_{1}\right)\left(y_{1}-x_{1} \mid .\right.
\end{aligned}
$$

Hence, $\operatorname{Lip}\left(\bar{A}_{0}\right) \leqslant \frac{5}{6}, \operatorname{Lip}\left(\bar{A}_{1}\right) \leqslant 1$ and $\operatorname{Lip}\left(\bar{A}_{2}\right) \leqslant \frac{5}{6}$. So in the case of $\tan \theta=\frac{1}{2}, \mathcal{F}$ is contractive with ratio not greater than $\left(\frac{5}{6}+1+\frac{5}{6}\right) / 3=\frac{8}{9}$. It follows from (6.10) that, for any $k$,

$$
\left|\gamma-\gamma_{k}\right| \leqslant 24\left(\frac{8}{9}\right)^{k}
$$

which implies that we can compute this Lyapunov exponent to any accuracy. By numerical computation, we obtain $\gamma=0.9793 \ldots$ and, for a.e. $b \in J_{\theta}$, $\operatorname{dim}_{\mathrm{H}} F_{\theta, b}=\operatorname{dim}_{\mathrm{B}} F_{\theta, b}=$ $\gamma / \log 3=0.8914 \ldots$ This typical value is strictly less than $\log 8 / \log 3-1=0.8927 \ldots$.

Remark 6.2. For $\tan \theta=1$,

$$
A_{0}=\left[\begin{array}{ll}
1 & 0 \\
2 & 2
\end{array}\right], \quad A_{1}=\left[\begin{array}{ll}
2 & 1 \\
1 & 2
\end{array}\right], \quad A_{2}=\left[\begin{array}{ll}
2 & 2 \\
0 & 1
\end{array}\right]
$$

Let $\delta(x, y)=\left|x_{1}-y_{1}\right|$. Then

$$
\operatorname{Lip}\left(\bar{A}_{0}\right) \leqslant \frac{1}{2}, \quad \operatorname{Lip}\left(\bar{A}_{1}\right) \leqslant \frac{1}{3}, \quad \operatorname{Lip}\left(\bar{A}_{2}\right) \leqslant \frac{1}{2},
$$

and thus $c \leqslant\left(\frac{1}{2}+\frac{1}{3}+\frac{1}{2}\right) / 3=\frac{4}{9}$. We have the error estimation

$$
\left|\gamma-\gamma_{k}\right| \leqslant(12)\left(\frac{4}{9}\right)^{k}
$$


We list some typical values of dimensions of sections as follows:

$$
\begin{aligned}
& \tan \theta=0: 0.8927 \ldots \quad(=\log 8 / \log 3-1), \\
& \tan \theta=1: 0.8858 \ldots, \\
& \tan \theta=\frac{1}{3}: 0.8926 \ldots, \\
& \tan \theta=\frac{1}{4}: 0.8917 \ldots
\end{aligned}
$$

According to the above numerical result, we pose the following conjecture.

Conjecture 6.3. If $\tan \theta \in \mathbb{Q}$ and $d_{\theta}$ is the typical value of $\operatorname{dim}_{H} F_{\theta, a}$ for almost all $a \in J_{\theta}$, then $d_{\theta}<(\log 8 / \log 3)-1$.

\section{Fractals like the Sierpinski carpet}

In fact, we can deal with the fractals like the Sierpinski carpet.

Given an integer $m \geqslant 2$, let $\left\{\psi_{i}\right\}_{i=1}^{k}$ be a family of different similitudes of $\mathbb{R}^{2}$ such that $\psi_{i}(x, y)=(x, y) / m+\left(c_{i}, d_{i}\right) / m$, where $c_{i}, d_{i} \in \mathbb{Z} \cap[0, m-1]$. Suppose that $E=\bigcup_{i=1}^{k} \psi_{i}(E)$ $\left(\subset[0,1]^{2}\right)$ is the self-similar set.

Fix $\theta$ and let $\left\{\tau_{j}: \mathbb{R} \rightarrow \mathbb{R}\right\}_{j=1}^{k}$ be linear mappings such that

$$
\psi_{j}^{-1}\left(L_{\theta, a}\right)=L_{\theta, \tau_{j}(a)} \text { for all } j .
$$

Write $\varsigma_{j}=\tau_{j}^{-1}, E_{\theta, a}=E \cap L_{\theta, a}$ and $\mathcal{J}_{\theta}=\left\{a: L_{\theta, a} \cap E \neq \varnothing\right\}$. Here $\left.\varsigma_{j}\right|_{\mathcal{J}_{\theta}}: \mathcal{J}_{\theta} \rightarrow \mathcal{J}_{\theta}$, since we have

$$
E_{\theta, \varsigma_{j}(a)} \supset \psi_{j}\left(E_{\theta, a}\right),
$$

which is like formula $(*)$.

Suppose that $\tan \theta=M / N>0$ is rational with $N, M \in \mathbb{N}$.

We make the assumption that $\mathcal{J}_{\theta}$ is an interval. For example, if the boundary of $[0,1]^{2}$ is contained in $E$, then $\mathcal{J}_{\theta}$ is an interval for each $\theta$. In fact, when the boundary $\partial[0,1]^{2}$ is contained in $\bigcup_{i=1}^{k} \psi_{i}\left([0,1]^{2}\right)$, e.g. the Sierpinski carpet, we have $\partial[0,1]^{2} \subset E$.

As in $\S 3$, there exist matrices $\mathcal{A}_{0}, \ldots, \mathcal{A}_{m-1}$, which are $(N+M) \times(N+M)$ nonnegative integer matrices. Because of the assumption, we can prove a lemma of ergodic type similar to Lemma 4.2. By using this lemma and formula (**), we can prove results similar to Propositions 4.3 and 4.4. As in $\S 5$, we also establish the equality for Hausdorff and box dimensions.

Consequently, a result like Theorem 1.1, for the fractals like the Sierpinski carpet, can be established when $\mathcal{J}_{\theta}$ is an interval.

Acknowledgements. This work was supported by the Morningside Center of Mathematics of Beijing, the National Science Foundation of China (grant nos 10241003, 10671180, 10501002 and 10301029) and the ZJNFS (grant no. 101026).

\section{References}

1. I. Benjamini And Y. Peres, On the Hausdorff dimension of fibres, Israel J. Math. 74 (1991), 267-279. 
2. H. Furstenberg, Disjointness in ergodic theory, minimal sets, and a problem in Diophantine approximation, Math. Systems Theory 1 (1967), 1-49.

3. J. HAWKes, Some algebraic properties of small sets, Q. J. Math. 26 (1975), 195-201.

4. J. E. Hutchinson, Fractals and self similarity, Indiana Univ. Math. J. 30 (1981), 713747 .

5. R. KENYON AND Y. PEREs, Intersecting random translates of invariant Cantor sets, Invent. Math. 104 (1991), 601-629.

6. J. F. LI, On the dimensions of intersections of Sierpinski carpet with lines, Master's thesis, Wuhan University (1997).

7. Q. H. Liu And Z. Y. Wen, On dimensions of multitype Moran sets, Math. Proc. Camb. Phil. Soc. 139(3) (2005), 541-553.

8. J. M. MARstrand, Some fundamental geometrical properties of plane sets of fractional dimensions, Proc. Lond. Math. Soc. 4 (1954), 257-302.

9. P. MatTila, Geometry of sets and measures in Euclidean spaces (Cambridge University Press, 1995).

10. R. D. Mauldin and S. C. Williams, Hausdorff dimension in graph directed constructions, Trans. Am. Math. Soc. 309(1-2) (1988), 811-839.

11. P. WALters, An introduction to ergodic theory (Springer, 1982). 